\title{
ANTIBACTERIAL SILICA NANOPARTICLES WITH PH-SENSITIVE RELEASE PROPERTIES AS FILLERS FOR DENTAL COMPOSITE MATERIALS
}

\author{
Hendrik Fullriede ${ }^{1}$, Nico Timpe ${ }^{2}$, Lothar Borchers ${ }^{3}$, Meike Stiesch ${ }^{3}$, Henning Menzel ${ }^{2}$, Peter Behrens ${ }^{1}$ \\ ${ }^{1}$ Institut für Anorganische Chemie, Leibniz Universität Hannover, Germany \\ ${ }^{2}$ Institut für Technische Chemie, Technische Universität Braunschweig, Germany \\ ${ }^{3}$ Klinik für Zahnärztliche Prothetik und Biomedizinische Werkstoffkunde, \\ Medizinische Hochschule Hannover, Germany \\ hendrik.fullriede@acb.uni-hannover.de
}

\begin{abstract}
Silica nanoparticles with nanopores were used to incorporate antimicrobial agents, like silver nanoparticles or chlorhexidine. By the functionalization of the particles with different organosilanes a controlled drug release is possible. A stimuli-response system was able to react to acidic conditions as caused by an infection and to release the drugs only when necessary. These particles could be incorporated into dental composite materials to prevent infections or periimplantitis.
\end{abstract}

Keywords: dental composites, silica nanoparticles, drug delivery, silver nanoparticles, stimuli-response

\section{Introduction}

Dental composites consist of an organic polymer matrix and inorganic filler particles. Commonly used fillers are spherical silica particles produced by flame pyrolysis which are nonporous and nonfunctionalized. Our approach is to use nanoporous silica nanoparticles (NPSNPs) synthesised via sol-gel chemistry. Within their pores, antimicrobial agents can be incorporated. For instance, silver nanoparticles exhibit very good antimicrobial properties ${ }^{[1]}$. As an organic drug, chlorhexidine (CHX), well established in dentistry, was employed. ${ }^{[2]}$ In this way, dental composite materials could be equipped with antibacterial properties to prevent biofilm formation and periimplantitis.

The surface of the silica particles can be functionalized by post-grafting to control the release kinetics. Anionic groups can enhance the interactions of the cationic $\mathrm{CHX}$ molecules with the silica particles. Poly(4-vinylpyridine) (PVP) can act as a stimuli-response functionalization (Fig. 1). At neutral pH values, the polymer chains are folded, capping the pores with the antimicrobial agent inside. When the $\mathrm{pH}$ value decreases, for example due to a bacterial infection, the nitrogen atoms of the pyridine groups become protonated and thus are positively charged. These positive charges repel each other and the polymer chains unfold and straighten, thus unblocking the pores. The drug can then be released and can combat the infection until the $\mathrm{pH}$ values return to a normal state.

In addition, it is possible to control the morphology of the silica particles during the synthesis. Anisotropic silicate nanoparticles were used to enhance the mechanical prop- erties of dental composites due to entanglement and bridging effects. ${ }^{[3]}$ Different shapes were tested to investigate the effect of the particle morphology on the mechanical properties of the dental composite materials.
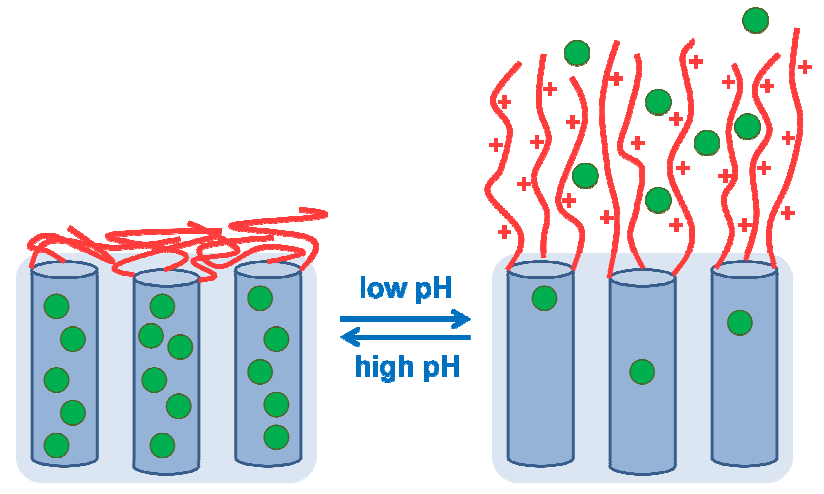

Figure 1: $\mathrm{pH}$-sensitive drug release.

\section{Methods}

Nanoporous silica nanoparticles were prepared via the sol-gel route from alkaline aqueous solutions. Tetraethoxysilane (TEOS) and 3-mercaptopropyltrimethoxysilane (MPTS) were used as silicon precursors, cetyltrimethylammonium bromide (CTAB) acted as structure-directing agent to build up the porous system. After the reaction, the particles were washed with ethanol and calcined at high temperatures to remove the organic surfactants. The morphology of the particles was determined by scanning electron microscopy (SEM) and the pore system was analyzed by argon sorption measurements. Modification of the particle surface with different trialkoxyorganosilanes was applied by post-grafting reactions carried out in toluene. Silver nanoparticles were generated inside the pores by thermal decomposition after soaking the particles in $\mathrm{AgNO}_{3}$ solution.

Composite materials were produced by photopolymerisation of bisphenol A glycidylmethacrylate (Bis-GMA) and triethylene glycoldimethacrylate (TEGMA), commonly used basic monomers for dental composites.

The release of silver ions from the silica particles or the silver-silica loaded composites was measured in different media by atomic absorption spectroscopy (AAS). Chlorhexidine was detected via UV-Vis spectroscopy. 


\section{Results}

SEM images reveal silica particles with a broad variation of morphologies, e.g. spheres, fibers or spirals. IR measurements show that both removal of the organic templates and different modification reactions were successful. Thiol, sulphate, carboxylic acid, amino, PVP and methacrylate groups could be grafted onto the surface. Ar sorption measurements show a high BET surface area and a narrow pore size distribution in the range of about $3 \mathrm{~nm}$. Silver nanoparticles could be generated within the pores of the silica particles. Silver ions were released from the silver nanoparticles over a period of several months. From the composite materials, the released concentrations were correspondingly smaller.

The antiseptic agent chlorhexidine was also introduced into the pores. Determination of the CHX release show an initial burst release and then a long term release, depending upon the modification of the silica particles. The PVP-functionalized silica particles show a significantly higher $\mathrm{CHX}$ release rate in acidic media compared to neutral $\mathrm{pH}$ values

\section{Discussion}

Nanoporous silica nanoparticles with spherical and anisotropic shape were successfully synthesized with a pore system suitable for drug delivery purposes. The surface of the particles was modified with various organic groups to control the release kinetics of $\mathrm{CHX}$. The functionalization with hydrophobic methacrylate groups enhances the dispersion of the fillers within the organic matrix.

One of the advantages of using silver nanoparticles located within the silica pores in combating bacterial infection is the long term release which could possibly prevent infections over a long period of time. Although the composite materials showed a smaller amount of $\mathrm{Ag}^{+}$ions released, depending on the ionic strength of the release medium the concentration could be increased.

CHX as an alternative antibacterial agent shows a burst release from NPSNPs and may thus reduce the number of bacteria in an effective way. PVP apparently blocks the pores under neutral conditions but allows release under acidic conditions, like during an infection. Thus, a $\mathrm{pH}-$ sensitive drug release system was constructed.

In vitro and in vivo testings of the different particles and the composites are necessary to confirm the antibacterial properties and their biocompatibility.

\section{Acknowledgement}

This work was supported by the DFG within the Collaborative Research Program SFB 599 "Sustainable bioresorbable and permanent implants based on metallic and ceramic materials" (TP D12).

\section{Bibliography}

[1] Gusmán, M G; Dille, J; Godet, Stephan; World Academy of Science, Engineering and Technology, 2008, 43, 357364.

[2] Carriljo, MR; Carvalho, RM; Sousa, EN; Nicolau, J; Breschi, L; Mazzoni, A; Tjäderhane, L; Tay, FR; Agee, K; Pashley, DH; Dent. Mater., 2010, 26, 779-785.

[3] Tian, M; Gao, Y; Liu, Y; Hedin, N. E; Fong, H; Dent. Mater., 2008, 24, 235-243. 